\title{
PATH INTEGRAL FOR THE DIRAC EQUATION
}

\author{
J. POLONYI \\ Laboratory of Theoretical Physics, Louis Pasteur University \\ 3 rue de l'Université 67087 Strasbourg, Cedex, France \\ and \\ Department of Atomic Physics, L. Eötvös University \\ Puskin u. 5-7 1088 Budapest, Hungary \\ E-mail: polonyi@fresnel.u-strasbg.fr
}

\begin{abstract}
A path integral representation is given for the solutions of the $3+1$ dimensional Dirac equation. The regularity of the trajectories, the non-relativistic limit and the semiclassical approximation are briefly mentioned.
\end{abstract}

There are different integral or sum representations for the solution of the Dirac equation. The summation over trajectories on $1+1$ dimensional lattice 1 can be generalized by the help of the Grassman variables 2 for $3+1$ dimensions 3. The method presented here⿴囗十 has the advantage that the integration is over the real trajectories in the continuous three-space, just as in the case of the non-relativistic Schrödinger equation. The disadvantage is that the spin and the chirality flips are still described by spinors so the integrand of the path integral consists of the product of $4 \times 4$ matrices. But this complication can be avoided and the c-number integration recovered by means of additional compact variables $\mathrm{E}$.

We start with massless chiral fermions whose equation of motion is $\left(\partial_{0}+\right.$ $c \partial \cdot \sigma) \phi=0$. The $\hbar$-independence reflects that the causality is maintained even on the level of the quantum fluctuations. We introduce the infinitesimal time propagator $G(x, y ; \Delta \tau)=e^{i \mathcal{A}(x, y ; \Delta \tau)}$ defined by

$$
\phi(x, \tau+\Delta \tau)=\int d y G(x, y ; \Delta \tau) \phi(y, \tau),
$$

where $\tau=t c$. The choice of the action $\mathcal{A}(x, y ; \Delta \tau)$ can be motivated in the following manner: (i) We expect a self reproducible propagator,

$$
e^{i \mathcal{A}\left(x, y ; \tau_{1}+\tau_{2}\right)}=\int d z e^{i \mathcal{A}\left(x, z ; \tau_{1}\right)} e^{i \mathcal{A}\left(z, y ; \tau_{2}\right)},
$$

what suggests a quadratic dependence of $\mathcal{A}$ in the coordinates. (ii) Translational invariance further restricts the propagator to

$$
\mathcal{A}(x, y ; \Delta \tau)=\frac{1}{2}(x-y)^{j} A_{j k}(\Delta \tau)(x-y)^{k}+(x-y)^{j} B_{j}(\Delta \tau) .
$$


(iii) Rotational symmetry imposes $A_{j k}=A \delta_{j, k}$ and that the vector $B_{j}$ should be parallel to the spin, $B_{j} \approx \sigma_{j}$. (iv) We want to keep the massless propagator $\hbar$-independent so the only choice is $A=a / \Delta \tau^{2}$ and $B_{j}=b \sigma_{j} / \Delta \tau$, where $a$ and $b$ are dimensionless constants. The substitution of this propagator into (11) shows that the desired equation of motion is recovered in the continuum limit, $\Delta \tau \rightarrow 0$ for $a=b$. The chiral amplitude $\phi(x)$ is thus evolved by the path integral

$$
\begin{aligned}
G(x, y ; \tau) & =\prod_{j}\left\{\mathcal{N}^{-1} \int d x_{j} e^{i \kappa\left[\frac{1}{2}\left(\frac{x-y}{\Delta \tau}\right)^{2}-\frac{x-y}{\Delta \tau} \cdot \sigma\right]}\right\} \\
& =\int D[x(t)] T\left\{e^{i \frac{\kappa}{\Delta \tau} \int d \tau\left[\frac{1}{2}\left(\frac{d x}{d \tau}\right)^{2}-\frac{d x}{d \tau} \cdot \sigma\right]}\right\},
\end{aligned}
$$

where we have taken $a=b=\kappa$. Notice the explicit linear divergence, $\Delta \tau^{-1}$, in the action, in analogy with Quantum Field Theory where the bare, regulated action contains the divergent coupling constants.

The finite time propagator in the Fourier and real spaces is

$$
\begin{aligned}
\tilde{G}(p, \tau) & =e^{-i \frac{3 \tau}{2 \Delta \tau}} e^{-i \tau\left(\Delta \tau \frac{1}{2 \kappa \hbar^{2}} p^{2}+\frac{1}{\hbar} p \cdot \sigma\right)}, \\
G(x, y ; \tau) & =\mathcal{N}^{-1} e^{i \kappa \frac{\tau}{\Delta \tau}\left(\frac{1}{2}\left(\frac{x-y}{\Delta \tau}\right)^{2}-\frac{x-y}{\Delta \tau} \cdot \sigma\right)} .
\end{aligned}
$$

The correct spectrum $E(p)= \pm|p|$ is recovered in the continuum limit according to $\tilde{G}(p, \tau)$. $G(x, y ; \tau)$ shows that whenever the particle wanders off the light cone the diverging phase of the exponent cancels the non-causal amplitude.

The equation of motion for massive fermions in the presence of an external potential $A_{\mu}(x), i \partial_{\tau} \psi=\left[\alpha \cdot(-i \partial+e / c \hbar A)+\beta / \lambda-e / c \hbar A_{0}\right] \psi$ where $\lambda=\hbar / m c$, is generated by the path integral

$$
G(x, y ; \tau)=\int D[x(t)] T\left\{e^{i \frac{\kappa}{\Delta \tau} \int d \tau\left[\frac{1}{2}\left(\frac{d x}{d \tau}\right)^{2}-\frac{d x}{d \tau} \cdot \alpha\right]} e^{i \int d \tau\left[\frac{e}{\hbar c} \gamma^{0} \not A-\beta / \lambda\right]}\right\},
$$

with $A=\gamma^{0} A_{0}+\gamma \cdot A$. One can show that the resulting propagator can be written for $A_{\mu}=0$ as

$$
G(x, y ; \tau)=\int \frac{d p}{(2 \pi \hbar)^{3}} U(p) e^{\frac{i}{\hbar} p \cdot(x-y)-\frac{i}{\hbar} \beta t m c^{2} \sqrt{1+\left(\frac{p}{m c}\right)^{2}}} U^{\dagger}(p),
$$

where $U(p)$ describes a basis transformation, so it reproduces the usual relativistic spectrum.

The non-relativistic limit appears as a crossover between the scaling laws

$$
<\left(\frac{\Delta x}{\Delta \tau}\right)^{2}>= \begin{cases}1 & \Delta \tau<<\lambda \\ \lambda / \Delta \tau & \Delta \tau>>\lambda\end{cases}
$$


at $\Delta \tau \approx \lambda$. This result can be made more plausible my noting that that the only dimensional parameter for the average velocity in the relativistic region where mass is negligible is $c$. Thus the trajectories of our path integral become smoother then their non-relativistic counterpart, suggesting that the fractallike propagation of the first quantized non-relativistic quantum mechanics can be embedded into a smoother, more "classical" relativistic quantum dynamics.

In order to obtain the saddle point approximation consider the amplitude

$$
\int D[x(t)] \eta_{f}^{\dagger} T\left\{e^{i \frac{\kappa}{\Delta \tau} \int d \tau\left[\frac{1}{2}\left(\frac{d x}{d \tau}\right)^{2}-\frac{d x}{d \tau} \cdot \alpha\right]} e^{i \int d \tau\left[\frac{e}{\hbar c} \gamma^{0} \not A-\beta / \lambda\right]}\right\} \eta_{i},
$$

where $\eta_{i}$ and $\eta_{f}$ are Dirac spinors. The saddle point is the solution of the equation

$$
\frac{\delta \text { integrand of eq. }(9)}{\delta x(\tau-\Delta \tau)}=0
$$

what can be written as

$$
\frac{x(\tau)+x(\tau-2 \Delta \tau)-2 x(\tau-\Delta \tau)}{\Delta \tau^{2}}=\frac{\eta_{f}^{\dagger} F(\tau) G_{0}(x, y ; \tau) \eta_{i}}{\eta_{f}^{\dagger} G_{0}(x, y ; \tau) \eta_{i}},
$$

where

$$
\begin{gathered}
F(\tau)=\frac{1}{\Delta \tau} U(\tau)\left[L(\tau) \cdot \alpha+\frac{\Delta \tau}{2} \partial \gamma^{0} \not A+S(\tau) T(\tau) \cdot \alpha U(\tau)\right. \\
-e^{i \Delta \tau\left(\gamma^{0} \not A-\beta / \lambda\right)}\left(L(\tau-\Delta \tau) \cdot \alpha-\frac{\Delta \tau}{2} U(\tau-\Delta \tau) \gamma^{0} \partial A U^{\dagger}(\tau-\Delta \tau)\right. \\
\left.\left.+S(\tau-\Delta \tau) T(\tau-\Delta \tau) \cdot \alpha U^{\dagger}(\tau-\Delta \tau)\right) e^{-i \Delta \tau\left(\gamma^{0} \not A-\beta / \lambda\right)}\right] U^{\dagger}(\tau) \\
U(\tau)=e^{-i \kappa \dot{x}(\tau) \cdot \alpha}, \quad \dot{x}(\tau)=\frac{x(\tau)-x(\tau-\Delta \tau)}{\Delta \tau} \\
L_{j k}(\tau)=\frac{\dot{x}_{j}(\tau) \dot{x}_{k}(\tau)}{x^{2}(\tau)}, \quad T_{j k}=\delta_{j k}-L_{j k} \\
S(\tau)=\frac{\sin (\kappa|\dot{x}(\tau)|)}{\kappa|\dot{x}(\tau)|}
\end{gathered}
$$

and $G_{0}$ is the integrand of (9) evaluated at the saddle point. We find that the saddle point trajectory depends on the initial and final spinors. Furthermore, the presence of the term $O\left(\dot{x}^{2}\right)$ in the action is needed for the recovery of the usual saddle point structure. In fact, the action $O(\dot{x})$ would yield one saddle 
point trajectory for each initial coordinate in contrast to the non-relativistic limit where the initial coordinate and velocity are needed to specify the saddle point. For a given initial coordinate $x(\tau)$ and velocity $\dot{x}(\tau+\Delta \tau)$ one can solve (11) for $x(\tau+2 \Delta \tau)$. The repetition of this procedure yields the saddle point trajectory corresponding to a given initial coordinate and velocity. Since the integration over real trajectories with a c-number integrand treats fermions in the same footing as bosons one can finally develop the same non-perturbative methods for both cases.

This is the point where we can fix the parameter $\kappa$ which does not influence the expectation values in the continuum limit. It is easy to see that the straight line trajectory satisfies (11) for $A=0$ when $\kappa=n \pi \tau /|x-y|$. Since the average velocity at the cutoff scale is $c$ for $\Delta \tau \rightarrow 0$ the choice $\kappa=\pi$ gives the renormalized path integral which is dominated by as smooth trajectories as possible.

Finally we mention that the term $O\left(\dot{x}^{2}\right)$ in the action is reminiscent of the Wilson term for lattice fermions because it suppresses the unwanted latticecopies. But it is given in the first quantized formalism and retains chiral invariance. Actually there is no problem of giving a space-time lattice regulated form of our path integral which avoids the Nielsen-Ninomiya no-go theorem 6 . The passage over the second quantized stage is quite a challenge. According to the spin-statistics theorem the phase factors corresponding to the rotation by $2 \pi$ and the exchange of two equivalent particles are the same. Since the former is built in our path integral formalism one expects to achieve the correct Green functions for fermions without the use of Grassman variables. Another interesting feature is that the term $O\left(\dot{x}^{2}\right)$ in the action should generate a chiral invariant Wilson term.

\section{References}

1. R. P. Feynman, A. R. Hibbs, Quantum Mechanics and Path Integrals, (McGraw-Hill, N.Y.), 1965; T. Jacobson, L. S. Schulman, J. of Phys. A 17, 375 (1984); B. Gaveau, T. Jacobson, M. Kac, L. S. Schulman, Phys. Rev. Lett. 53, 419 (1984).

2. B. Gaveau, L. S. Schulman, Phys. Rev. D 36, 1135 (1987); T. Miura, Progr. of Th. Phys. 61, 1521 (1979); D. M. Gitman, S. I. Zlatev, Phys. Rev. D 55, 7701 (1997).

3. See L. Schulman's contribution to this Proceedings.

4. P. Gosselin, J. Polonyi, Ann. Phys. 267, (1998).

5. Work in progress.

6. H. B. Nielsen, M. Ninomiya, Nucl. Phys. 185, 20 (1981). 